\title{
PAT: waking up a lazy sleeping beauty
}

\author{
Xiangdong $\mathrm{Fu}^{1}$, Jianru Zuo ${ }^{1}$ \\ ${ }^{I}$ National Center for Plant Gene Research, Institute of Genetics and Developmental Biology, Chinese Academy of Sciences, Beijing \\ 100101, China.xdfu@genetics.ac.cn
}

Cell Research (2007) 17:402-410. doi:10.1038/cr.2007.42; published online 14 May 2007

Once upon a time, there was an unusual corn; and unlike its normal siblings, its stalk grew towards the ground rather than in an upward direction. The sleeping stature of the corn was portrayed in early 1930s as "lazy" by Jenkins and Gerhardt [1]. Later, lazy appeared to be a fashion in the plant kingdom, found in rice, barley, tomato and several other species [2]. These seemingly magic spellchanted plants have drawn considerable interests of plant biologists and breeders for many decades. To breeders, the lazy phenotype, viewed as tillering or branching angles of stems (or termed as culms in crop plants), represents a key characteristic important for controlling photosynthesis efficiency and planting density, and thus a crucial factor in determining crop production [6]. Previous physiological studies suggested that the prostrate or lazy phenotype was attributed, at least in part, to the loss of gravitropism. For example, the rice lazy 1 plants are ageotropic, whereas wild type (WT) plants are negatively geotropic [2].

Gravity plays a major role in plant morphogenesis by determining the directional growth of plant organs. Upon germination, shoots grow upward, capturing light, and by contrast, roots penetrate the soil and grow downward, taking up water and nutrients. Plant tropisms have fascinated biologists for at least the last two centuries [3]. The classical Cholodny-Went theory proposes that gravity-induced auxin (a plant hormone) redistribution across a gravistimulated plant organ is responsible for the gravitropic response [4]. A unique feature of the auxin action is characterized by the polar auxin transport (PAT) mechanism, involving two classes of specific proteins known as influx and efflux carriers [5], which allows for a finely-tuned distribution of auxin in planta, thereby properly regulating a variety of cellular activities. Over the past two decades, significant progress has been made in understanding the action of auxin and tropic growth of primary roots in Arabidopsis thaliana. When presented with an alteration in the relative direction of the gravitational vector, the direction of root growth is changed, resulting in re-alignment of the direction of growth to that parallel with the new vector. In the gravitropic response, the peripheral reverse flow is diverted via redistribution of the cellular location of auxin efflux carriers [5], leading to the differential auxin accumulation in the underside lateral root cap cells of gravitationally stimulated roots [6]. The resultant auxin gradient is thought to extend to the distally located elongation region of the root, where relatively higher levels of auxin on the underside of the root inhibit cell growth.

Gravitropism is a key characteristic controlling photosynthesis efficiency and planting density, one of the most important agronomic traits in determining crop production [7]. Thus the lazy phenotype has attracted much attention of the breeders. Previous physiological studies on lazy mutants consistently pointed to a potential relationship with auxin actions [8], however, the precise mechanism by which the auxin gradient affects gravitropic responses in crop remains unknown. A direct link between the rice gravitropic responses and the PAT-mediated auxin redistribution is now revealed by Jiayang Li and colleagues in this issue of Cell Research [9]. Li et al. present convincing evidence showing that agravitropism in the rice lazyl (la1) mutant is caused by an impaired PAT. The lal mutation renders the endogenous auxin, as monitored by a specific reporter gene DR5-GUS [10], to distribute in an enlarged domain that somehow has moved downward to the basal part of the mutant coleoptiles. Consistent with the above observation, in a PAT assay, basipetal transport, but not acropetal transport, of radioactive indolyl-3-acetic acid (IAA, a major bioactive auxin) in etiolated coleoptiles was substantially elevated in the lal mutant compared to that in wild type. Moreover, the gravity-stimulated lateral IAA transport between the lower and upper halves of the coleoptiles was also significantly impaired in the lal cole- 
optiles. Thus, $L A 1$ is involved in the regulation of the PAT function to mediated distribution of IAA, through which it mediated the gravitropic responses in rice.

The long struggling history of studies on crop gravitropism was partly owing to the lack of molecular characterization of $L A Z Y$ genes. Unfortunately, the rice $L A 1$ gene is located near the centromere in Chromosome 11, a region difficult for genetic mapping due to the very low frequency of genetic recombination. Nevertheless, the $L A 1$ gene was identified by Li et al. [9] through a tremendous effort by monitoring genetic recombination from over $30000 \mathrm{~F}_{2}$ mutant plants. $L A 1$ appears to be a grass-specific gene with no apparent homologs found in dicots. This raises an intriguing question: do dicots and monocots utilize distinctive mechanisms in responding to gravitropic signals? The $L A 1$ gene encodes a protein of unknown function, which contains a putative transmembrane domain (TMD) and a putative nuclear localization signal (NLS). LA1 was shown to localize in both the plasma membrane and the nucleus in a TMD- and NLS-dependent manner. This unique subcellular localization pattern suggests possible shuttling of the protein between the plasma membrane and the nucleus under different physiological conditions. Interestingly, transgenic studies showed that the strength of the mutant phenotype was negatively correlated with the $L A 1$ expression level, strongly suggesting that $L A 1$ acts as a negative regulator of PAT and thus the tiller angles during development.

A new life started when the Sleeping Beauty was woken up. The discovery made by Li et al. [9] resolves a long-lasting puzzle and represents a major breakthrough in the understanding of the cellular and molecular basis of gravitropism in crop plants. This study opens up new prospects for understanding the gravitropism in monocot plants, and therefore will feed plant biologists' appetites for many years. Not the least, crop-selection programs should benefit.

\section{Reference}

1 Jenkins M, Gerhardt F. A gene influencing the composition of the culm in maize. Iowa Ag Exp Sta Research Bull 1931; 138:121151.

2 Jones JW, Adair CR. A "lazy" mutation in rice. J Heredity 1938; 28:315-318.

3 Darwin C, Darwin F. The Power of Movement in Plants. London: John Murray, 1880.

4 Went FW, Thimann KV, eds. Phytohormones. New York: Macmillan, 1937.

5 Friml J, Wisniewska J, Benkova E, et al. Lateral relocation of auxin efflux regulator PIN3 mediates tropism in Arabidopsis. Nature 2002; 415:806-809.

6 Ottenschläger I, Wolff P, Wolverton C, et al. Gravity-regulated differential auxin transport from columella to lateral root caps cells. Proc Natl Acad Sci USA 2003; 100:2987-2991.

7 Li Z, Andrew HP, Shannon RMP, James WS. RFLP facilitated analysis of tiller and leaf angles in rice (Oryza sativa L.). Euphytica 1999; 109:79-84.

8 Godbole H, Takahashi H, Hertel R. The lazy mutation in rice affects a step between statoliths and gravity-induced lateral auxin transport. Plant biol 1999; 1:379-381.

9 Li P, Wang W, Qian Q, et al. LAZY1 controls rice shoot gravitropism through regulating polar auxin transport. Cell Res 2007; 17:402-410.

10 Ulmasov T, Murfett J, Hagen G, Guilfoyle TJ. Aux/IAA proteins repress expression of reporter genes containing natural and highly active synthetic auxin response elements. Plant Cell 1997; 9:1963-1971. 